\title{
Wirelessly controllable inflated electroactive polymer (EAP) reflectors
}

\author{
Xiaoqi Bao, Yoseph Bar-Cohen, Zensheu Chang, Stewart Sherrit and Mircea Badescu \\ Jet Propulsion Laboratory, California Institute of Technology, 4800 Oak Grove Drive, \\ Pasadena, CA, USA 91109-8099
}

\begin{abstract}
Inflatable membrane reflectors are attractive for deployable, large aperture, lightweight optical and microwave systems in micro-gravity space environment. However, any fabrication flaw or temperature variation may results in significant aberration of the surface. Even for a perfectly fabricated inflatable membrane mirror with uniform thickness, theory shows it will form a Hencky curve surface rather than the desired parabolic or spherical surface. Precision control of the surface shape of extremely flexible membrane structures is a critical challenge for the success of this technology. Wirelessly controllable inflated reflectors made of electroactive polymers (EAP) are proposed in this paper. A finite element model was configured to predict the behavior of the inflatable EAP membranes under pre-strains, pressures and distributed electric charges on the surface. To explore the controllability of the inflatable EAP reflectors, an iteration algorithm was developed to find the required applied electric field distribution for correcting the aberration of a Hencky curve to the desired parabolic curve. The correction capability of the reflectors with available EAP materials was explored numerically and is presented in this paper.
\end{abstract}

Keyword: Electroactive polymer, EAP, inflatable membrane mirror, thin film antenna, controllable reflector.

\section{INTRODUCTION}

Inflatable membrane reflectors i.e. visible or infrared optical mirrors, electromagnetic wave antennas, are attractive for large-apertures optical and microwave systems operating in micro-gravity space because of their ultra-lightweight and deployablity features. Inflated membrane reflectors usually consist of two circular membranes that are sealed on their edges, attached to a tensioning ring and inflated to a pressure to produce the desired curved surface. These inflated reflectors have been used for various applications where low imaging acuity is sufficient. The main problem that prohibits the use of these reflectors in high quality imaging system is the difficulty in controlling their shape to the desired paraboloid or spherical curvatures. Even for a perfectly fabricated uniform membrane, Inflation will generate a surface with a non spherical or parabolic profile called a Hencky curve [Marker et al 1998]. The aberration of the Hencky curve from the parabolic is termed "W-curve" following its shape. Although the aberration can be reduced by increasing the pre-strain in the membrane, the correction is limited. An example of the aberration is shown in Fig. 11 and 12 where half of the $\mathrm{W}$-curve, from center to the edge, is presented. The maximum value is $6 \mathrm{~mm}$ for a $2-\mathrm{m}$ diameter, $1.25-\mathrm{m}$ focal length, $1 \%$ pre-strained inflated reflector. Other approaches such as non-uniform thickness membrane were also proposed to correct the aberration [Meinel et al 1999]. However, the fabrication is difficult for precision variable thickness control. Precision control of the surface shape of extremely flexible membrane structures is a critical challenge for the success of this technology [Jenkins et al 1998, Wangneret al 2000, Martin et al 2000, Dimkov et al 2000]. It requires matched soft actuation materials. Electroactive polymers (EAP) are one of potential candidates of the actuation materials [Bar-Cohen (ed.) 2001]. The material is soft and usually fabricated in form of thin film. The EAP materials will be used as a study material to investigate the controllability of the membrane using wireless actuation to correct for the aberrations.

The EAP materials can be categorized in two main classes. One is the ionic EAP [Onishi et al 2000, Bao et al 2002]. The ionic EAP's contain electrolyte in a polymer frame. They show large bending deformation at low voltage excitation. However, the actuation mechanism of these materials involves transport of ions and molecules from one side of the film to the other. The component and properties of the electrolyte have to be well maintained to keep the performance stable. This type EAP may not be a good candidate for space application due to the possible volatility of the electrolyte. Another class is electric field EAP [Costen et al 2001, Cheng et al 2001, Zhang et al 2002, Bao et al 2004]. It includes piezoelectric, electrostrictive, ferroelectric or dielectric polymers. These materials behave like

1 xbao@jpl.nasa.gov; fax 818-393-4057 
capacitors under excitation voltages and the strain is usually proportional to the square of the applied voltage. Although they require relative high voltage $(10 \sim 100 \mathrm{~V} / \mu \mathrm{m})$ to create maximum strain, these polymers are dry and have relativelystable performances. The study presented in this paper focuses on electric field EAP's. The electrically induced strain of these EAP's can be up to 10 percent or higher. An example is a P(VDF-TrFE)-based Co/Ter-polymer having a maximum transverse strain of $\sim 4 \%$ [Cheng et al 2001, Bao, 2004].

In a previously published paper [Bao et al, 2003], we propose a reflector made of a single-layer EAP and electrically controlled by electron scanning beam. The concept reflector is illustrated in Fig. 1. The back surface could be electrodeless to allow a continuous voltage distribution for smooth control. Polymers can hold charge on their surface due to the high surface and bulk resistivity. This property is illustrated in the drum of laser printers where the charge is maintained to reproduce the image. However, it is still expected that the charge will decay slowly due to the limited resistivity of the polymer. An EAP with relatively large resistivity can be selected to reduce the discharge rate and the electron gun can re-scan the surface to compensate the charge loss.

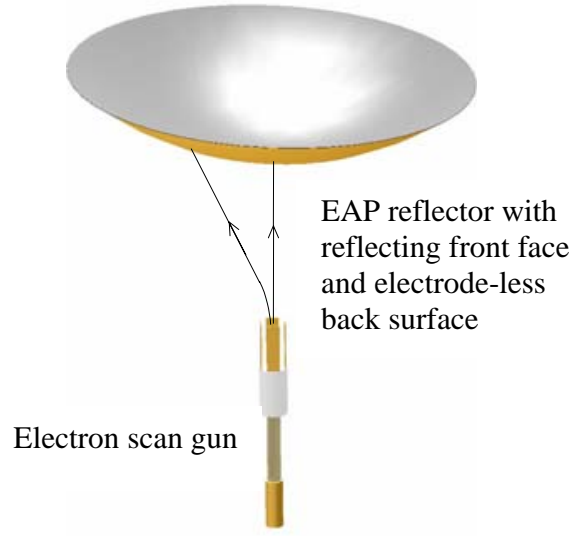

Fig. 1 The concept of a wirelessly controllable EAP mirror

We also suggested a shape control method based on the effect of electrically introducing non-uniform extension in the EAP and numerically proved that a distributed voltage can form a parabolic mirror of $f / 0.625$ from an initially planar EAP membrane with maximum electrically induced strain of $4 \%$.

In order to control the reflector to the required precision, a close-loop control system including a sensor to detect the shape of the reflector, an adaptive controller and the controllable reflector outlined above are required. The adaptive controller can tolerate the performance variation of the elements in the loop. The control precision of such a system will, for the most degree, depend on the precision of the sensor. A controllable secondary reflector or lens may be added to the observation system to compensate remaining errors of the main reflector.

Although we numerically proved the EAP membrane can be controlled to desired papraboloid surface without the help of inflation, the inflation will prove the membrane reflectors extra rigidity and stability. In the following sections, the controllability of the EAP in inflatable reflectors is investigated. A numerical iteration algorithm was developed to find the control voltage distribution for approaching the desired shape. The effort is focused on the correction of the aberration of the Hencky curve to the parabolic curve.

\section{CONTROL THE ABERRATION OF INFLATED REFLECTORS BY EAP ACTUATION}

A numerical iteration algorithm was developed to find the required electrical voltage distribution to correct the aberration. For the inflatable reflectors made of EAP, voltage applied to a certain area will tend to expand the area and 
help the pressure to move the area and the surrounding area towards the pressure direction. The iteration algorithm is based on this physical understanding. We calculate the aberration of the membrane from the desired surface first and, then, add a small electrical voltage with a distribution proportional to the aberration to the EAP membrane in each step. Then, we recalculate the aberration and iterate the procedure. A flowchart of the iteration is shown in Fig. 2.

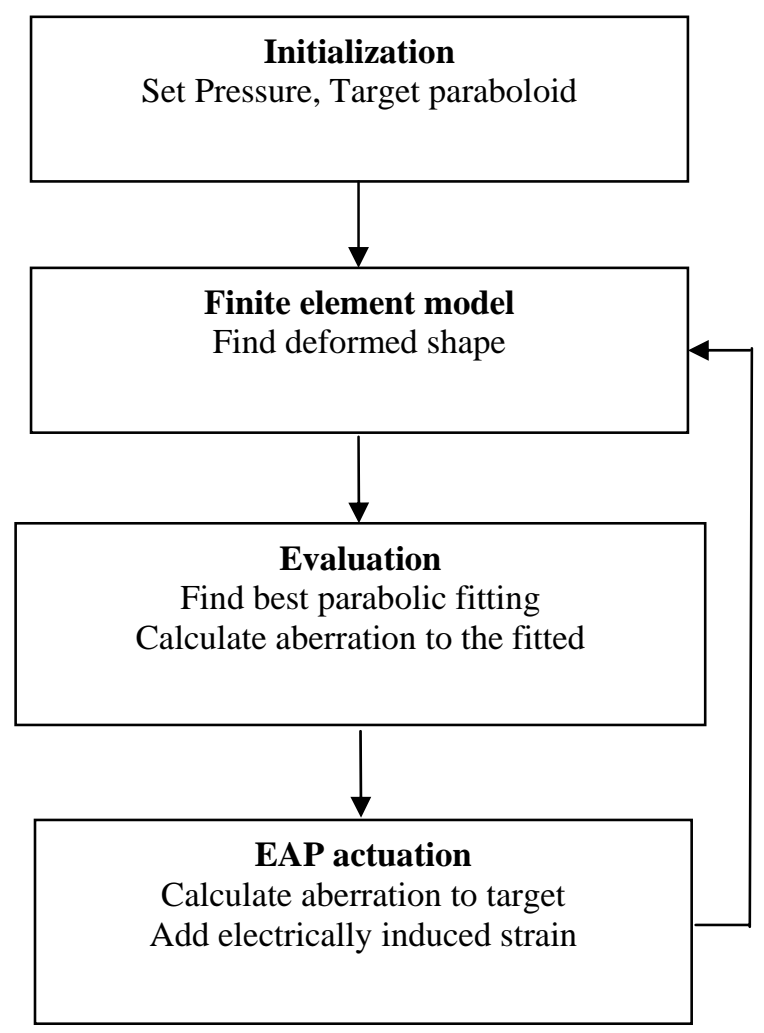

Fig. 2 Flowchart of the numerical iteration algorism

A finite element software package ANSYS was used to calculate the shape of the membranes under pressure, the applied electrical voltage on the EAP and possible pre-strain. The geometry and material properties of the membrane used in the computation are listed in Table 1. The material properties are determined referring of the P(VDF-TrFE)based Co/Ter-polymer that has a maximum transverse strain of $4 \%$ [Cheng et al 2001, Bao et al 2004]. The planar films were expressed by 100 shell elements (Shell-151, ANSYS). The element describes the shell and a surface. Therefore the deformation of the surface of the membrane due to the thickness change is not taken in account. The deformation is in the range of a few micrometers for the $10 \mu \mathrm{m}$ thick membrane. This error can be compensated if high accuracy is required. The extension strain/stress created by the electric voltage on the EAP membrane was simulated by thermal expansion by setting proper material thermal coefficients and a corresponding temperature change. "Large Deflection" function of the ANSYS was activated to take the nonlinear effect of geometry into account.

Table 1. Parameters used in computation

\begin{tabular}{|c|c|c|c|}
\hline Diameter $(\mathrm{m})$ & Thickness $(\mu \mathrm{m})$ & E $(\mathrm{Gpa})$ & Poisson's ratio $\sigma$ \\
\hline 2 & 10 & 1 & 0.3 \\
\hline
\end{tabular}

This algorithm was first verified using the case for which analytical solution is known and available [Bao et al 2003]. It is to form a parabolic reflector from a planar EAP membrane purely by electric actuation with no pressure. The results are shown in Fig. 3-6. Figure 3 shows the analytical solution of the required electrically induced strain in order to 
curve the membrane. The corresponding voltage distribution can be calculated easily for EAP with known properties. For typical electric field EAP, the electrically induced strain is proportional to the square of applied voltage as

$$
S=\alpha V^{2}
$$

and the required voltage is as

$$
V=\sqrt{\frac{S}{\alpha}}
$$

where the $S$ is the electrically induced strain, $V$ is the voltage, and $\alpha$ is the material constant.

Fig. 4 shows the aberration of the computed shape from the best fit parabolic curve. The parabolic curve was in the form of

$$
z=a r^{2}+b .
$$

and the coefficients $a$ and $b$ were found by a least square curve fitting method. This aberration in the level of several micrometers is caused by the limitation of the accuracy of the finite element model, i.e. limited element number, convergence error in large deflection calculations etc. Fig. 5 and 6 present the corresponding results found by the iteration algorithm. The number of iterations was 50 . The aberration obtained by the iteration algorithm is in the same range as the finite element result based on the analytical solution. It was concluded that the iteration algorithm worked well for this case.

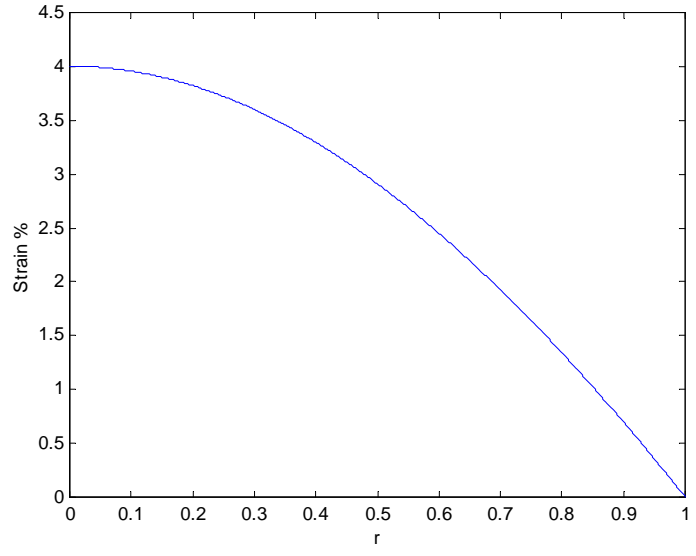

Fig. 3 Analytical solution of required strain distribution for $\mathrm{z}=0.2 \mathrm{r}^{2}$ membrane reflector

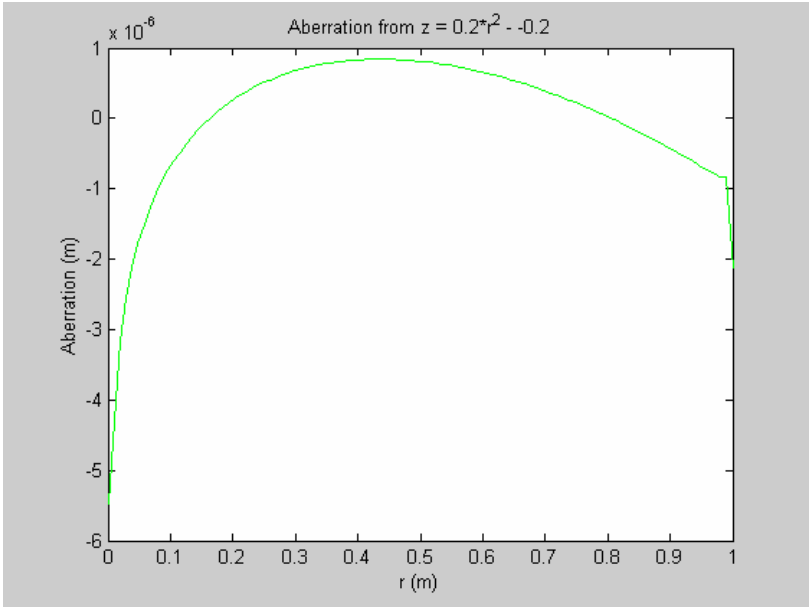

Fig. 4 Aberration of the membrane shape computed by FE using analytical solution to the best fitted parabolic curve. 


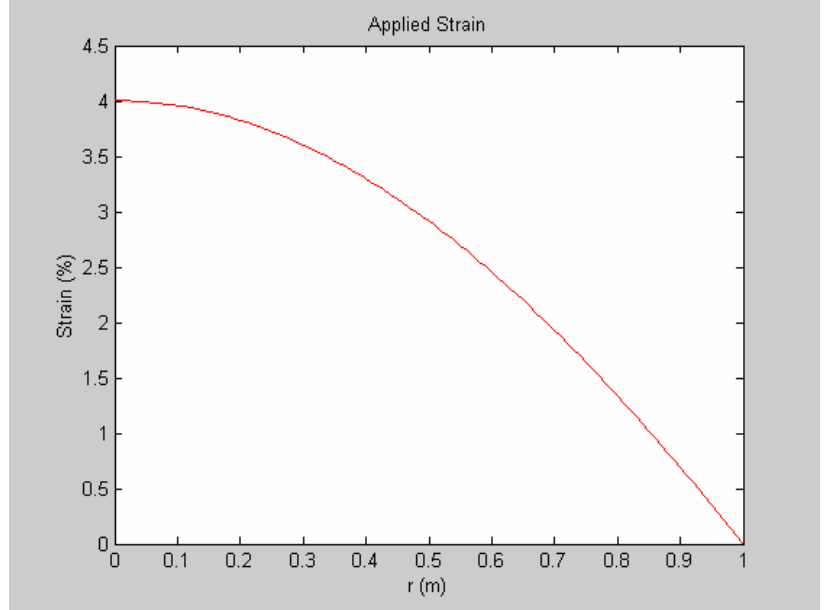

Fig. 5 Numerical solution of required strain distribution for $\mathrm{z}=0.2 \mathrm{r}^{2}$ membrane reflector by iteration algorism.

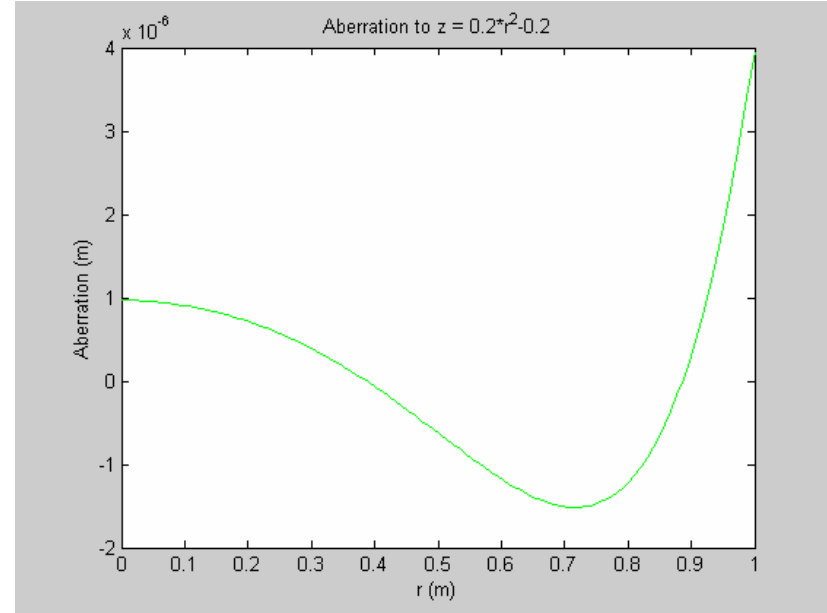

Fig. 6 Aberration of the membrane shape computed by FE using the numerical solution of the iteration algorism to the best fitted parabolic.

The algorithm was applied to inflatable EAP membranes. We set a paraboloid surface as a target surface. The computation started with a pressure $\mathrm{P}$ under which the deformation of the membrane is less than the target parabolic curve. We looked for the required electrical actuation using the algorithm. However, in the iteration the added electrically induced strains at the edge are always zero because both the target surface and the calculated membrane are fixed at the edge. For the previous verification case, the zero is just the right solution at the edge as the analytical solution showed. However, it may not be correct when the pressure is applied. To overcome the problem, modifications of the algorithm are needed. Trial computation showed that the required strain jumped at the edge because the algorithm constrains the application of the electrically induced strain at the edge. A reasonable guess is that the strain should be extended to the edge. We found the required strains are always decreasing as the edge is approache.. A modification of the algorithm was undertaken to set the strain in the zone outside of the minimum strain ring to be the minimum value.

This modification may lead to negative required strains in some areas. Negative strains mean to shrink the membrane but the EAP actuation is always to extend the area. To overcome the difficulty, a pre-strain in the membrane by stretching the membrane is necessary. The pre-strain can be set to equal to the minimum of the required strain found by the iterations. After adding the pre-strain, the required electrically induced strains become all positive or zero.

Several different cases were computed. Results of these examples are shown in the Fig. 7-10. The target profile of the parabolic surface was set as $\mathrm{z}=0.2 \mathrm{r}^{2}-0.2$ and the diameter was $2 \mathrm{~m}$. The pressure applied was $1000 \mathrm{~Pa}$. In the iteration, we monitored the maximum aberrations of the whole surface and that of the area within $90 \%$ of the diameter. After 380 iterations, the aberration of the area within $90 \%$ of the diameter converged to a few micrometers as shown in Fig. 7. The aberration of the whole area converged to a few micrometers after 950 iterations. The remaining aberration is within the range of computation errors. An improvement of the computing accuracy may improve the results. As is shown in Fig. 9 and in the enlarged curve in Fig. 10, the large aberration is located in the narrow rings near the edge and the center. The aberration in most area is much smaller, less than $0.1 \mu \mathrm{m}$. Shadowing the ring areas or coating them with absorbing layers should greatly improve the quality of the reflector. This result proves that, in principle, the Henkey-curve aberration of the inflatable reflectors can be corrected electrically when the reflectors are made of EAP membranes.

The required electrically induced strain is $-1.06 \%$ at the edge as is shown in Fig. 8 . This means that a $1.06 \%$ prestrain is needed to shift the required electrically induced strain to be positive. With the pre-strain, the maximum of the required electrically induced strain is $4.4 \%$. It is actually a little higher than the required $4 \%$ for form the same paraboloid surface without inflation pressure (see Fig. 3-6). The benefit of the inflated reflectors is the applied pressure will make the reflectors more rigid and stable. It should be noticed that, for illustrating the capability of the available 
EAP materials, the reflector calculated as an example has a very short focal length. The f-number of the reflector is only 0.625. For most of applications, the required reflectors are much flatter than this example and require less electrically induced strain for the aberration correction.

For comparison, the aberration of a reflector with the same parameters but without EAP actuation control was computed. The reflector has the same geometry and pre-strain. The pressure was adjusted to $3900 \mathrm{~Pa}$ and resulted in a curved profile having a best fit of $\mathrm{z}=0.1961 \mathrm{r}^{2}-0.2026$, which is close to the target profile. The result is presented in Fig. 11 and 12. The maximum aberration is more than $6 \mathrm{~mm}$, which is much larger than the controlled EAP membrane and not acceptable for most imaging system.

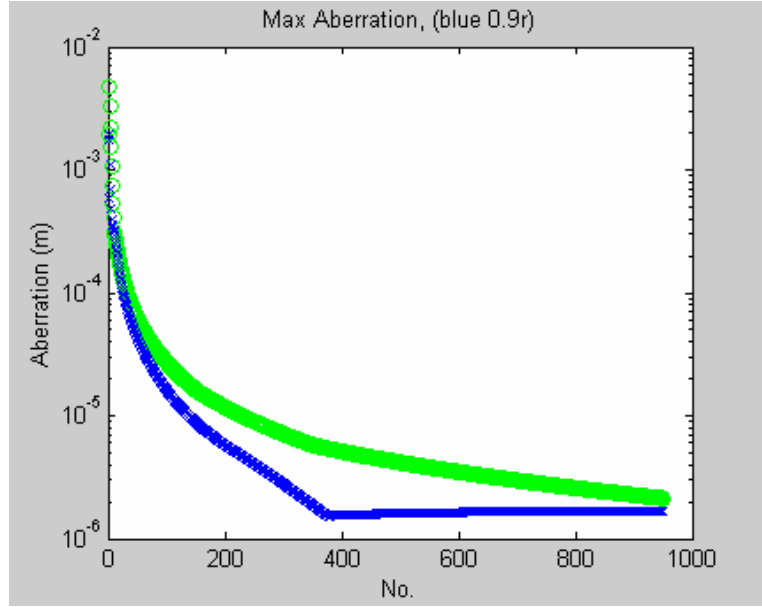

Fig. 7 The maximum aberration of the computed membrane shape vs. iteration number. Up line is that on whole surface, the low line is that within $r$ $=0.9 \mathrm{~m}$.

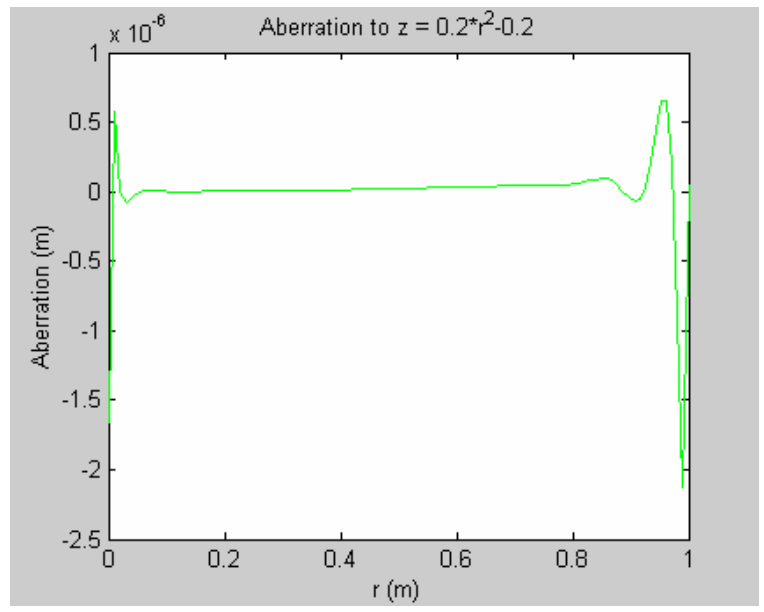

Fig. 9 The computed remaining aberration of the membrane after applying control actuation

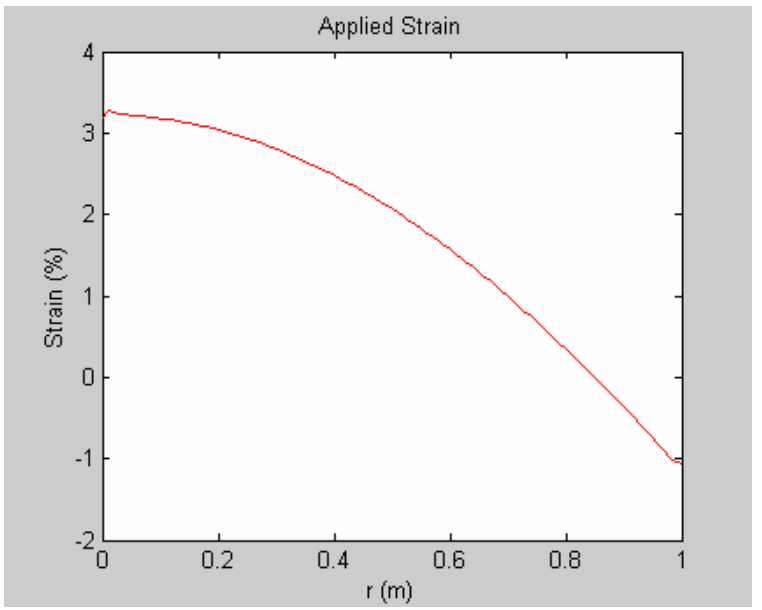

Fig. 8 The required electrically-induced strain computed by numerical iteration for aberration correction.

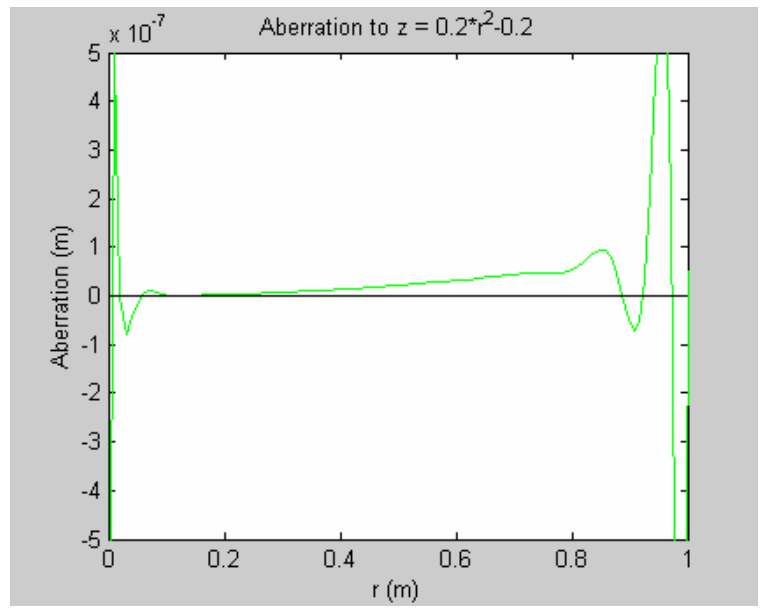

Fig. 10 Enlarged remaining aberration of the membrane after applying control actuation 


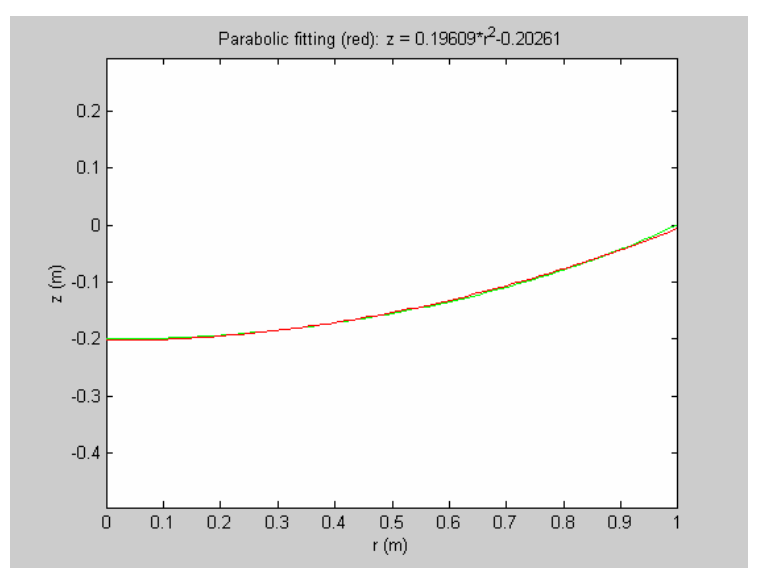

Fig. 11 The computed membrane (gray) with $1.06 \%$ pre-strain under applied pressure of 3900 $\mathrm{Pa}$ and the best fitted parabolic curve (black).

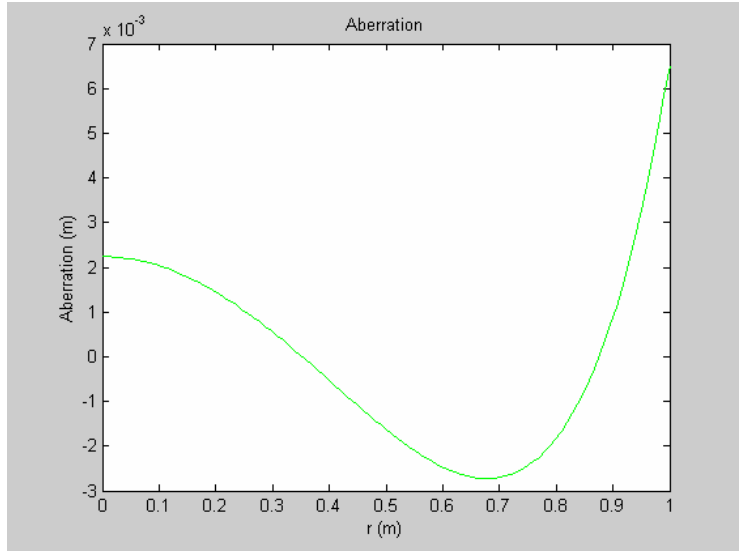

Fig. 12 The aberration of the membrane with $1.06 \%$ pre-strain under applied pressure of 3900 $\mathrm{Pa}$ to the best fitted parabolic curve.

\section{CONCLUSIONS}

Controllable inflatable reflectors made of single-layer EAP membrane were proposed. Applying a distributed voltage to the backside of the EAP reflector can control the shape of the reflector. The voltage may be applied wirelessly by using electron scanning beam.

An iteration algorithm based on a physical understanding of the mechanism was developed to find the required electric field distribution to obtain the desired surface shape. The algorithm was applied to correct a common aberration of inflatable reflectors. Computed results show that the proposed EAP membrane reflector is able to change the profile of the inflated membranes to the desired parabolic curve electrically. The results show that the aberration can therefore be corrected.

\section{ACKNOWLEDGEMENTS}

The reported research at Jet Propulsion Laboratory (JPL), California Institute of Technology, was carried out under a contract with National Aeronautics Space Agency (NASA).

\section{REFERENCES}

Bao X., Bar-Cohen Y., Chang Z., Sherrit S., "Numerical modeling of single-layer electroactive polymer mirrors for space applications,” Proceedings of SPIE - The International Society for Optical Engineering, v 5051, 2003, p 381-388

Bao X., Bar-Cohen Y., Chang Z., Sherrit S., “Characterization of bending EAP beam actuator,” Proceedings of the SPIE Smart Structures and Materials Symposium, San Diego, CA, 2004

Bao X., Y. Bar-cohen, S. Lih, "Measurements and macro models of ionomeric polymer-metal composites (IPMC)," Proc. of SPIE, v 4695, p 220-227, 2002.

Bar-Cohen, Y.(Ed.), Electroactive Polymer (EAP) Actuators as Artificial Muscles - Reality, Potential and Challenges, pp. 1-671, SPIE Press, Vol. PM98, 2001.

Bar-Cohen Y., X. Bao, S. Sherrit, S. Lih, "Characterization of the electromechanical properties of Ionomeric Polymer-Metal Composite (IPMC),” Proc. of SPIE, v 4695, p 286-293, 2002. 
Cheng Z.-Y., H.S. Xu, T. Mail, M. Chung, Q.M. Zhang, R.Y. Ting, "P(VDF-TrFE)-based electrostrictive Co/Terpolymers and its device performance,” Proc. of SPIE, v 4329, p 106-116, 2001.

Costen R.C., J. Su, J.S. Harrison, "Model for bending actuators that use electrostrictive graft elastomers,” Proc. of SPIE, v 4329, p 437-444, 2001.

Dimkov S.A., B.V. Kislitsyn, "Mathematical model of a variable-curvature thin-film mirror,” J. Opt. Technol. v 67, p 229-234, 2000.

Jenkins C.H., D.K. Marker, “Surface precision of inflatable membrane reflectors,” J. Solar Energy Eng. ASME, v 120, p 298-305, 1998.

Marker D., et al, “Optical evaluation of membrane mirrors with curvature,” SPIE V3430, 202-208(1998)

Martin J. W., et al, “Distributed sensing and shape control of piezoelectric bimorph mirrors,” J. Intell. Mater. Sys. Struc. v 11, p 744-757, 2000.

Meinel, Aden B.; Meinel, Marjorie; Aden B.;Breckinridge, J. B., "Inflatable Membrane Mirrors for Optical Passband Imagery,” NASA Ultra lightweight space optical challenge workshop, 24-25, March (1999)

Onishi K., S. Sewa, K. Asaka, N. Fujiwara, K. Oguro, "Morphology of electrodes and bending response of the polymer electrolyte actuator,” Electrochimica Act v 46, p 737-743, 2000.

Wangner J.W., G.S. Agnes, E. Magee, “Optical metrology of adaptive membrane mirrors,” J. Intell. Mater. Sys. Struc. v 11, pp 837-847, 2000.

Zhang Q.M., H. Li, M. Poh, F. Xia, Z. -Y. Cheng, H. Xu, C. Huang, “An all-organic composite actuator material with high dielectric constant,” Nature, v 419, p 284-287, 2002. 